\title{
Operational hub cells: a morpho-physiologically diverse class of GABAergic neurons united by a common function Rosa Cossart
}

\begin{abstract}
GABAergic microcircuits structure the activation of neuronal ensembles that support most cortical computations. Because of the heterogeneous nature of the GABAergic cell community, a full understanding of structure-function relationships in these microcircuits may be hampered by a reductionist approach that consists of classifying them according to an exhaustive collection of parameters. It therefore could be beneficial to our understanding of these complex cells to also consider other approaches. Thus, graph theory has recently taught us that biological networks often include hub nodes that are essential for information flow, and ensuing experimental evidence has demonstrated the existence of 'operational' hub neurons. So far, only GABAergic neurons have been identified as 'operational hubs', further emphasizing their critical function in controlling cortical network dynamics.
\end{abstract}

\section{Addresses}

INMED, INSERM U901, Aix-Marseille Université, Parc Scientifique de Luminy, BP.13, 13273 Marseille cedex 9, France

Corresponding author: Cossart, Rosa (rosa.cossart@inserm.fr)

\section{Introduction}

The coordinated activation of neuronal ensembles structured within networks is proposed to support the ongoing computations of the cerebral cortex in health and disease. Within these networks of neurons, GABAergic cells are key nodes that shape neuronal dynamics in space and time. This major network function is mirrored in the bewildering diversity of cortical GABAergic cells, which can be subdivided into many anatomically, physiologically, biochemically, and ontogenically distinct classes. One way to understand how GABAergic networks are organized to support cortical function and dynamics has been to attempt to classify them into distinct families based on various parameters [1]. The increasing accessibility and detail of morphological, molecular, physiological, and developmental data are both providing a substrate for this classification and encouraging GABAergic neuron taxonomy. It is undeniable that detailed characterization and classification have provided valuable information regarding GABA neuron function. However, it may well be that as our knowledge about the morphophysiological and genetic determinants of individual GABAergic cells increases, the more complex it becomes to reconcile the overall functional features of the system as a whole. Indeed, the problem of classifying and naming GABAergic neurons in the cerebral cortex has yet to reach a satisfactory consensus and single-cell diversity can blur our understanding of GABAergic networks. In addition, neurons belonging to different classes could potentially serve overlapping functions, thus providing cortical networks with a valuable redundancy that protects them, for example, from pathological insults.

The understanding of the functional organization of cortical GABAergic networks may thus be partially constrained by this reductionist approach and could benefit from the progress network science is currently experiencing [2]. Hence, after an initial description of cortical GABAergic microcircuits merely based on anatomical features and later taking into account multiple parameters to group them into distinct families using, for example, multiparametric clustering methods, it would be advantageous to know whether their functional organization, as a network, could be related to any previously described canonical structure (Figure 1). Indeed, many real networks, as different as the brain and the Internet, share similar architectures independently of their function. This universality is encouraging researchers from different disciplines to feed their research with concepts from network theory, and the recent finding of a morphophysiologically heterogeneous class of GABAergic cells unified by a common hub function supports this initiative $\left[3^{\bullet \bullet}\right]$.

\section{What is an 'operational' hub neuron? From theory to experimental evidence}

The term hub originates from the theoretical field of complex networks and clearly deserves some definition to avoid confusion, particularly in the context of experiments [2]. One of the most commonly described properties of a network is its degree distribution. It is used to distinguish between different types of network organization. The degree of a node is defined as its total number of links or connections to other nodes in the network. A connection does not refer to an absolute measure but to a parameter that is chosen during the analysis depending upon the type of network being studied and the questions being asked. Neuronal networks are mainly described by 


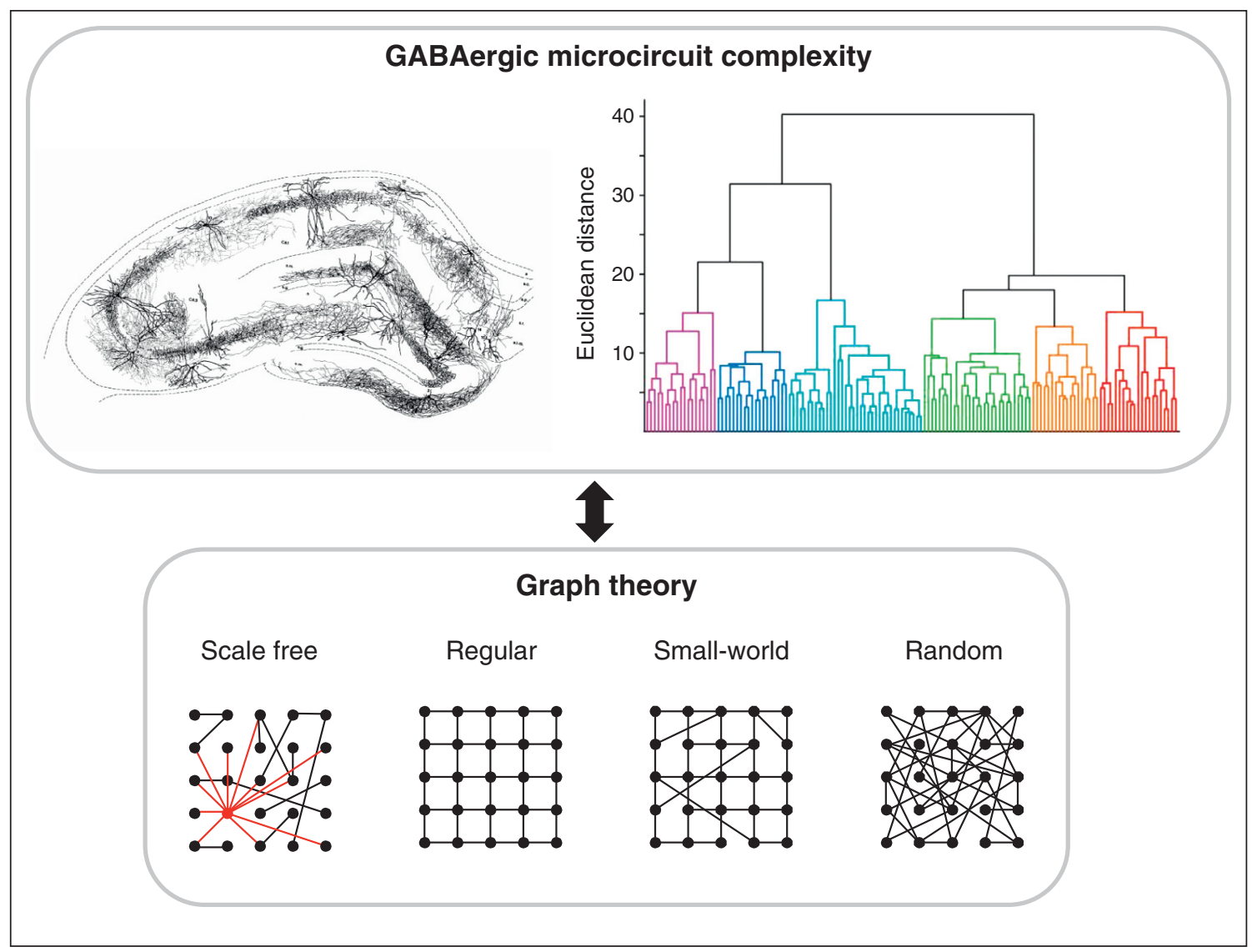

Understanding the functional organization of GABAergic microcircuits: from single-cell classifications (top) to network level analysis derived from graph theory (bottom). Anatomical description has provided valuable information regarding GABAergic neuron diversity as illustrated by the drawing taken with permission from [18]. More recent work combined a description of developmental, electrophysiological, and molecular properties of GABAergic neurons and applied cluster analyses to separate them into distinct subfamilies as illustrated by the dendrogram taken with permission from [25\%]. The functional organization of GABAergic cells, as a network, may be related to a previously described canonical structure as schematized below.

three different types of connectivity reflecting three parallel levels of investigation: anatomical (i.e. physical connection between two neurons), functional (statistical relationship between the activity of two neurons) and effective (causal relationship between the activity of two neurons).

Functional, effective, or anatomical hubs are neurons that have a high number of functional, effective, or anatomical connections to other neurons in networks where most neurons have very few. Experimentally speaking, we would like to introduce the term 'operational' hub neuron: a cell not only displaying a high functional connectivity degree (i.e. a functional hub), but also actively involved in generating network synchronization, the stimulation of which single-handedly impacts network dynamics. Experimental evidence for the existence of 'operational hub' neurons relied on the mapping of 'functional connectivity' in living brain slices based on the analysis of multineuron calcium activity $\left[3^{\bullet \bullet}\right]$.
Functional hub neurons were found to be a mixture of glutamatergic (presumably early-born [4], see below) and GABAergic cells [3 ${ }^{\bullet \bullet}$. However, electrophysiological recordings showed that only GABAergic neurons combined a high degree of functional, effective, and anatomical connectivity and acted as 'operational' hubs: their stimulation causally triggered the activation of many other neurons while disrupting spontaneous network oscillations.

It is easy to confuse hubs with GABAergic short-cuts between physically remote brain areas (such as the hippocampus and the medial septum [5,6] or the entorhinal cortex $\left[7,8^{\bullet}\right]$ ) for several reasons. First, both GABAergic hub cells and short-cuts are atypical network components with an extended axonal arborization unlike most other cortical GABAergic neurons. Secondly, in network space, short-cuts are nodes displaying long-range contacts (with 'length' referring to the number of functional links that separate nodes and not to their actual physical distance 


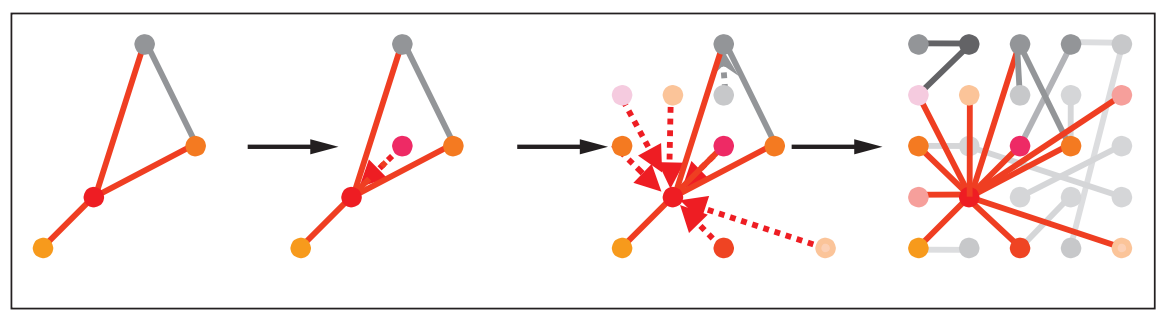

Scheme illustrating how scale-free networks grow following a preferential attachment rule derived from a 'homophilic' growth process. The dark red node, which turns into a hub, was present at the early stages of network growth and maximizes similarity with other nodes (similarity is schematized by the color of the nodes).

[2]), thus allowing information flow quickly. Hence, hub cells and GABAergic short-cuts likely function in coordinating network oscillations [9,10]. Further contributing to the confusion was the recent demonstration that a subpopulation of developmentally active GABAergic hub cells matures into short-cuts connecting the hippocampus to remote areas in the adult mouse $\left[11^{\circ}\right]$. Still, short-cuts that connect two distant regions through sparse synaptic targets do not necessarily display the high connectivity degree that characterizes hub cells.

\section{Is being a hub neuron a privilege of elite members of the GABAergic aristocracy [12]?}

As mentioned above, neurons that display a high functional connectivity degree in the developing hippocampus form a heterogeneous population that gathers both glutamatergic pyramidal neurons and GABAergic cells. Interestingly, two genetic fate mapping studies indicate that this distinct group of cells may share an early birthdate $\left[4,11^{\circ}\right]$. Thus some kind of 'droit d'aînesse', the old rule of inheritance whereby monarchy descends to the firstborn in preference to siblings, may also apply to neurons. This phenomenon may be the direct consequence of the 'preferential attachment rule' (Figure 2) that theoretically governs the growth of scalefree networks $\left[13^{\circ}\right]$. In addition, since there is also a 'temporal matching rule' by which cells tend to connect according to their temporal embryonic origin $\left[14^{\circ}\right]$, high connectivity glutamatergic and GABAergic neurons may well form a 'rich club' [15] sharing a high functional network impact, further reinforcing the privileges of that aristocracy.

However, in the developing hippocampus, only GABAergic neurons were experimentally shown to act as 'operational hubs'. In contrast, high connectivity glutamatergic neurons operate as assemblies, capable of triggering network synchronization when activated collectively when fast GABAergic transmission is impaired [4]. This raises the question of whether only GABAergic neurons can act as 'operational' hub cells. So far, experiments providing the existence of 'operational hub' cells have only been performed in the developing hippocampus [ $3^{\bullet \bullet}$. Since we now know about certain intrinsic and functional properties of developmental hub cells, it is tempting to speculate whether other previously described subtypes of GABAergic cells may also be part of the hub cell family. An example within the hippocampus is Cajal Retzius cells which are early generated and target extrahippocampal regions, in particular the entorhinal cortex [16]. Similarly, basket cells of the adult hippocampus may serve as 'operational hubs' since they are the site of high input convergence and output divergence [17-20] and are able to time the incidence of sharp waves recorded in adult hippocampal slices $\left[21^{\circ}\right]$. Similarly, enkephalin-positive CA1 GABAergic neurons are a small neuronal population that targets exclusively other interneurons in the subiculum and may thus coordinate network oscillations between that region and the hippocampus [22].

\section{Are hub neurons a specific subtype of GABAergic cell?}

The above conjectures naturally raise the question of whether hippocampal 'operational hubs' comprise a finite and well defined morpho-physiological variety of GABAergic cells. Let us focus on GABAergic cells with a hub function in the developing CA3 hippocampus. In this restricted particular case two families of 'operational hubs' could be distinguished based on their anatomical connectivity (Figure 3):

(1) 'connector hubs' [23] that display a long axon spanning across regions with sparse collaterals often invading the fimbria and comprising long-range projecting GABAergic neurons $\left[3^{\bullet \bullet}, 11^{\bullet}\right]$. Morphological heterogeneity prevails in this population, consistent with previous descriptions of GABAergic projection hippocampal neurons $[5,22]$. Some, if not all of these 'connector hubs' have an early temporal embryonic origin $\left[11^{\circ}\right]$ and express a variety of neurochemical markers. Function wise, the activation of connector hubs in the immature CA3 generally disrupts network synchronization, even 


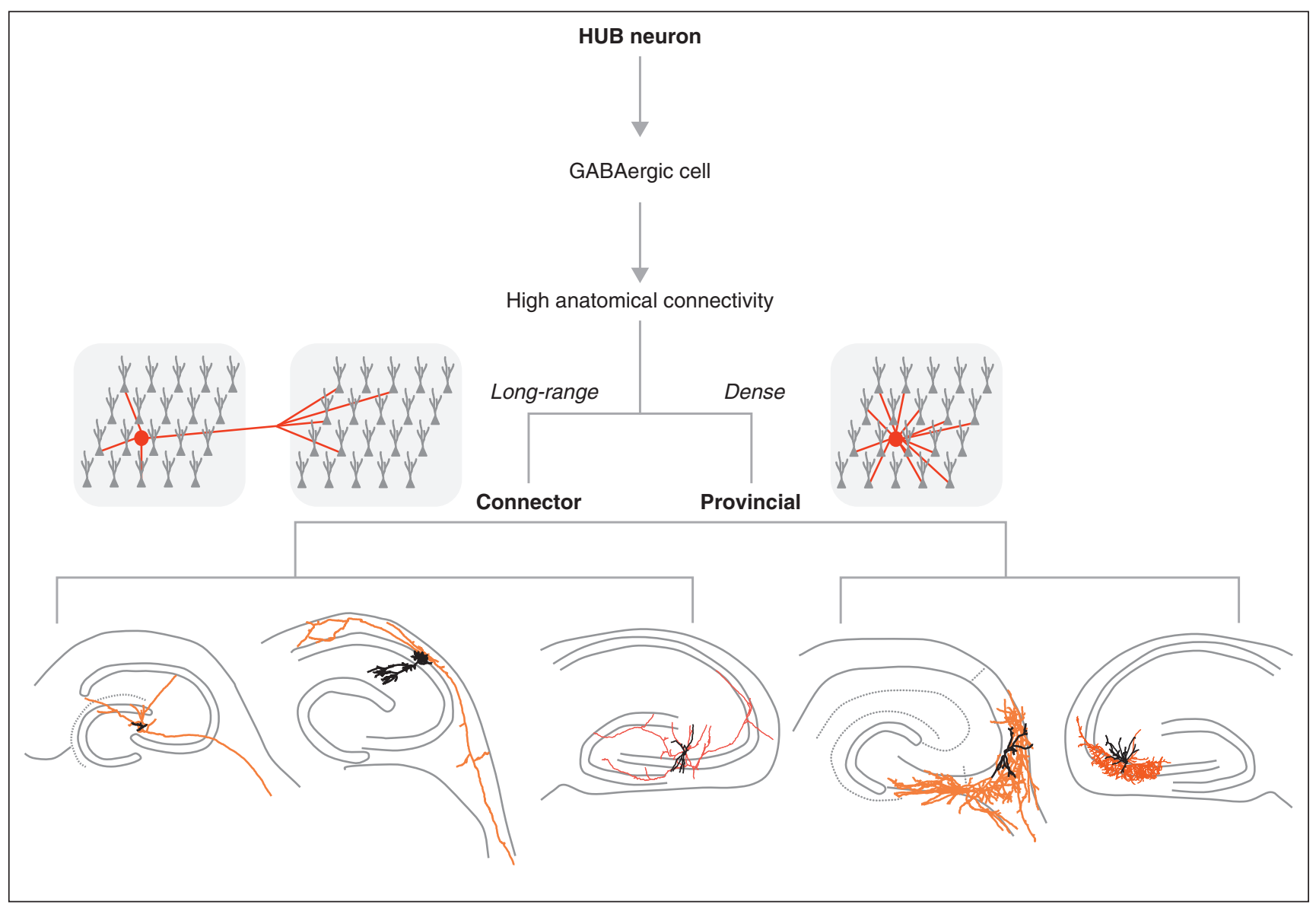

Diversity of operational GABAergic hub neurons in the hippocampus. Two families of 'operational hubs' in the developing CA3 could be distinguished based on their axonal connectivity patterns: 'connector hubs' that display a long axon comprising long-range projecting GABAergic neurons and 'provincial hubs', displaying a dense local axonal arborization with a 'basket-like' morphology. These two populations gather anatomically diverse cells as seen from the Neurolucida reconstructions of various hub cells filled during the experiments (illustrations taken with permission from [ $3^{\circ}$ ] and [11 $\left.{ }^{\circ}\right]$ ).

though these cells are recruited at the onset of GDPs $\left[3^{\bullet \bullet}, 11^{\bullet}\right]$. Interestingly, these cells have been recently shown to also critically determine the onset of epileptiform discharges induced by low $\mathrm{Mg}^{2+}$ conditions in the intact immature septohippocampal formation by directly synchronizing other interneurons $\left[24^{\circ \bullet}\right]$.

(2) 'provincial hubs' that are comprised of basket-like neurons that get connected within their own field through a dense but more local arborization pattern; their phasic activation may trigger network synchronization in the developing CA3 [ $\left.3^{\bullet \bullet}\right]$. The embryonic origin and adult fate of these basket-like hub neurons still remain to be determined. Although these cells may form a functionally distinct subclass of perisomatic neurons, diversity is also very likely to be present in this interneuron category [18], as suggested

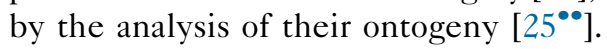

The level of heterogeneity observed in the 'operational hub' cell population so far may be the direct consequence of a developmental process by which hub neurons are not only the oldest neurons, but also those neurons that share a maximum number of features with other cells (Figure 2). This supposition is supported by a recent theoretical prediction. In order to explain the emergence of 'preferential attachment', the rule mentioned above by which scale-free networks grow and hub neurons emerge $\left[13^{\bullet \bullet}\right]$, it has been proposed that networks may grow following an homophilic process by which new nodes will preferentially connect to nodes that are similar to them $\left[26^{\circ \bullet}\right]$. In other words, similar types of neurons will preferentially connect to each other and therefore hub neurons should be cells that maximize some similarity index (Figure 2) and that way would incorporate the characteristics of many other neurons. This prediction is in agreement with various biological observations, 
including the fact that adult GABAergic neurons of the same subfamily tend to preferentially connect to each other via gap junctions [27], that cortical glutamatergic cells with the same target area are also more likely to connect to each other [28] or that clonally related neurons of the neocortex are more likely to be electrically [29], then synaptically connected [30].

Of course, the circuit components that account for the possible similarity index used by developing cortical networks remain an open question. In light of the GABAergic interneuron diversity, it will be of most interest to investigate whether this similarity index is derived from cellular intrinsic factors, extrinsic network determinants or both. Anyway, the similarity index must exert one of its most direct and extreme influence in the case of GABAergic hub cells. Hence, the fact that GABAergic projection neurons function as 'operational hub' cells may well be the direct consequence of that similarity rule since a GABAergic hippocampal neuron that targets extrahippocampal areas is on a connectivity level closer to a glutamatergic pyramidal neuron (i.e. projection neurons) than to any other subtype of GABAergic neuron.

\section{GABAergic hub neurons in pathology}

The issue of hub neuron function becomes particularly relevant in the context of pathologies for several reasons. First, as GABAergic hub neurons have been shown to synchronize early network oscillations, they should be essential for the proper development of circuits and an abnormal maturation of these cells may lead to developmental brain disorders. Second, a number of brain pathologies are associated with aberrant network oscillations as in the case of epilepsy. In fact, modeling work suggests that epileptic activity may depend on the existence of highly anatomically connected excitatory hub cells [ $\left.31^{\bullet \bullet}\right]$. However, recent experimental data obtained from slices using a chronic mouse model of temporal lobe epilepsy [32], from cultured rat slices [33] as well as from humans with focal epilepsy $[34,35]$, indicate variable paths of epileptiform synchronization dynamics. This raises the intriguing possibility that pathological and physiological network dynamics could be discriminated based on the stability of their spatial components and makes it less probable that the same 'operational hub' neuron repeatedly recruits or transmits information to similar assemblies in the epileptic synchronization process. Still, it was shown in acute models of epileptiform synchronization that specific GABAergic neurons acting as synchronization 'drivers' were critically involved in the generation of aberrant network bursts $\left[24^{\bullet \bullet}, 36\right]$. Therefore the involvement of 'operational hub' cells in pathology still remains an open question.

\section{Conclusion}

GABAergic 'operational hub' cells are a major functional subtype of hippocampal neurons. They represent a small but heterogeneous fraction of the total neuronal population, sharing a high functional and anatomical connectivity and most likely an early birthdate. In fact, hub cells may be designed to share as many characteristics as possible to a maximum number of other cell types including their glutamatergic counterparts, turning them into projection neurons and contributing to their striking diversity. We reason that being a hub is a privilege of neurons involved in GABAergic transmission in order to preserve network stability, but a different situation may prevail in pathological conditions. So far, 'operational hub' cells were found in the developing hippocampus, but given the presence of long-range projecting GABAergic cells in other structures, it may well be that this cell population is universally present and forms a 'rich club' supporting brain function, during development as well as later in health and disease.

\section{Acknowledgements}

I thank Sarah Feldt Muldoon, Vitor Sousa and Agnès Baude for helpful discussions and suggestions. I thank the FP7-ERC 'GABA Networks' grant (\# 242842), the Fondation pour la Recherche Medicale, The BettencourtSchueller Foundation, the C.N.R.S. and the I.N.S.E.R.M. for financial support.

\section{References and recommended reading}

Papers of particular interest, published within the period of review, have been highlighted as:

- of special interest

- of outstanding interest

1. DeFelipe J, Lopez-Cruz PL, Benavides-Piccione R, Bielza C, Larranaga $P$, Anderson S, Burkhalter A, Cauli B, Fairen A, Feldmeyer D, Fishell G, Fitzpatrick D, Freund TF, GonzalezBurgos G, Hestrin S, Hill S, Hof PR, Huang J, Jones EG, Kawaguchi $Y$, Kisvarday Z, Kubota $Y$, Lewis DA, Marin O, Markram H, McBain CJ, Meyer HS, Monyer H, Nelson SB Rockland K, Rossier J, Rubenstein JL, Rudy B, Scanziani M, Shepherd GM, Sherwood CC, Staiger JF, Tamas G, Thomson A, Wang Y, Yuste R, Ascoli GA: New insights into the classification and nomenclature of cortical GABAergic interneurons. Nat Rev Neurosci 2013, 14:202-216.

2. Feldt S, Bonifazi P, Cossart R: Dissecting functional connectivity of neuronal microcircuits: experimental and theoretical insights. Trends Neurosci 2011, 34:225-236.

3. Bonifazi P, Goldin M, Picardo MA, Jorquera I, Cattani A,

- Bianconi G, Represa A, Ben-Ari Y, Cossart R: GABAergic hub neurons orchestrate synchrony in developing hippocampal networks. Science 2009, 326:1419-1424.

The first experimental evidence for the existence of 'operational hub' neurons. This study was performed in the developing hippocampus using a combination of imaging and electrophysiology approaches.

4. Marissal T, Bonifazi P, Picardo MA, Nardou R, Petit LF, Baude A, Fishell GJ, Ben-Ari Y, Cossart R: Pioneer glutamatergic cells develop into a morpho-functionally distinct population in the juvenile CA3 hippocampus. Nat Commun 2012, 3:1316.

5. Jinno S, Klausberger T, Marton LF, Dalezios Y, Roberts JD, Fuentealba P, Bushong EA, Henze D, Buzsaki G, Somogyi P: Neuronal diversity in GABAergic long-range projections from the hippocampus. J Neurosci 2007, 27:8790-8804.

6. Shinoda K, Tohyama M, Shiotani Y: Hippocampofugal gammaaminobutyric acid (GABA)-containing neuron system in the rat: a study using a double-labeling method that combines retrograde tracing and immunocytochemistry. Brain Res 1987, 409:181-186.

7. Ino T, Matsuzaki S, Shinonaga $Y$, Ohishi H, Ogawa-Meguro R, Mizuno N: Direct projections of non-pyramidal neurons of 
Ammon's horn to the amygdala and the entorhinal cortex. Neurosci Lett 1990, 115:161-166.

8. Melzer S, Michael M, Caputi A, Eliava M, Fuchs EC

- Whittington MA, Monyer H: Long-range-projecting GABAergic neurons modulate inhibition in hippocampus and entorhinal cortex. Science 2012, 335:1506-1510.

Functional characterization of long-range GABAergic short-cuts between the hippocampus and the medial entorhinal cortex using optogenetic tools.

9. Buzsaki G, Geisler C, Henze DA, Wang XJ: Interneuron diversity series: circuit complexity and axon wiring economy of cortical interneurons. Trends Neurosci 2004, 27:186-193.

10. Caputi A, Melzer S, Michael M, Monyer $\mathrm{H}$ : The long and short of GABAergic neurons. Curr Opin Neurobiol 2013, 23:179-186.

11. Picardo MA, Guigue P, Bonifazi P, Batista-Brito R, Allene C,

- Ribas A, Fishell G, Baude A, Cossart R: GABA pioneer cells comprise a subpopulation of hub neurons in the developing hippocampus. Neuron 2011, 71:695-709.

Fate-mapping study showing that hippocampal GABAergic hub neurons include early born GABAergic cells originating from the earliest stages of neurogenesis and that develop into Sst-containing interneurons with an extrahippocampal projection.

12. Case M, Soltesz I: Discreet charm of the GABAergic bourgeoisie: superconnected cells conduct developmental symphonies. Neuron 2009, 64:780-782.

13. Barabasi $A L$, Albert R: Emergence of scaling in random - networks. Science 1999, 286:509-512

A theoretical paper demonstrating that scale-free networks grow following a 'preferential attachment' rule whereby new nodes tend to preferentially connect to the more connected ones.

14. Deguchi Y, Donato F, Galimberti I, Cabuy E, Caroni P: Temporally

- matched subpopulations of selectively interconnected principal neurons in the hippocampus. Nat Neurosci 2011, 14:495-504.

A study showing that hippocampal principal neurons tend to form microcircuits defined by their distinct neurogenesis time windows and distinct patterns of gene expression.

15. van den Heuvel MP, Sporns O: Rich-club organization of the human connectome. J Neurosci 2011, 31:15775-15786.

16. Ceranik K, Deng J, Heimrich B, Lubke J, Zhao S, Forster E, Frotscher M: Hippocampal Cajal-Retzius cells project to the entorhinal cortex: retrograde tracing and intracellular labelling studies. Eur J Neurosci 1999, 11:4278-4290.

17. Sik A, Tamamaki N, Freund TF: Complete axon arborization of a single CA3 pyramidal cell in the rat hippocampus, and its relationship with postsynaptic parvalbumin-containing interneurons. Eur J Neurosci 1993, 5:1719-1728.

18. Freund TF, Buzsáki G: Interneurons of the hippocampus. Hippocampus 1996, 6:347-470.

19. Tukker JJ, Lasztoczi B, Katona L, Roberts JD, Pissadaki EK, Dalezios Y, Marton L, Zhang L, Klausberger T, Somogyi P: Distinct dendritic arborization and in vivo firing patterns of parvalbumin-expressing basket cells in the hippocampal area CA3. J Neurosci 2013, 33:6809-6825.

20. Sik A, Penttonen M, Ylinen A, Buzsaki G: Hippocampal CA1 interneurons: an in vivo intracellular labeling study. J Neurosci 1995, 15:6651-6665

21. Ellender TJ, Nissen W, Colgin LL, Mann EO, Paulsen O: Priming of - hippocampal population bursts by individual perisomatictargeting interneurons. J Neurosci 2010, 30:5979-5991.

A study showing that stimulating a subset of hippocampal perisomatic cells was able to single-handedly initiate sharp wave population bursts in vitro.

22. Fuentealba $P$, Tomioka $R$, Dalezios $Y$, Marton LF, Studer $M$, Rockland K, Klausberger T, Somogyi P: Rhythmically active enkephalin-expressing GABAergic cells in the CA1 area of the hippocampus project to the subiculum and preferentially innervate interneurons. J Neurosci 2008, 28:10017-10022.

23. Bullmore E, Sporns O: Complex brain networks: graph theoretical analysis of structural and functional systems. Nat Rev Neurosci 2009, 10:186-198.

24. Quilichini PP, Le Van QM, Ivanov A, Turner DA, Carabalona A

- Gozlan H, Esclapez M, Bernard C: GABA Hub neurons mediate gamma-frequency oscillations at ictal-like event onset in the immature hippocampus. Neuron 2012, 74:57-64.

A study showing that a few hub-like GABAergic neurons that project to the septum are instrumental for the generation of gamma frequency oscillations occurring at the onset of epileptiform discharges generated pharmacologically in the intact septohippocampal formation.

25. Tricoire L, Pelkey KA, Erkkila BE, Jeffries BW, Yuan X, McBain CJ:

- A blueprint for the spatiotemporal origins of mouse hippocampal interneuron diversity. J Neurosci 2011 , 31:10948-10970.

The first comprehensive fate-mapping study of the spatio-temporal origins of hippocampal GABAergic neurons.

26. Papadopoulos F, Kitsak M, Serrano MA, Boguna M, Krioukov D:

- Popularity versus similarity in growing networks. Nature 2012 489:537-540.

A theoretical paper showing that preferential attachment is a phenomenon that can emerge from a growth process that ensures the maximization of a similarity index.

27. Hestrin S, Galarreta M: Electrical synapses define networks of neocortical GABAergic neurons. Trends Neurosci 2005, 28:304-309.

28. Brown SP, Hestrin S: Cell-type identity: a key to unlocking the function of neocortical circuits. Curr Opin Neurobiol 2009, 19:415-421.

29. Yu YC, He S, Chen S, Fu Y, Brown KN, Yao XH, Ma J, Gao KP Sosinsky GE, Huang K, Shi SH: Preferential electrical coupling regulates neocortical lineage-dependent microcircuit assembly. Nature 2012, 486:113-117.

30. Yu YC, Bultje RS, Wang XQ, Shi SH: Specific synapses develop preferentially among sister excitatory neurons in the neocortex. Nature 2009, 458:501-504.

31. Morgan RJ, Soltesz I: Nonrandom connectivity of the epileptic

- dentate gyrus predicts a major role for neuronal hubs in seizures. Proc Natl Acad Sci U S A 2008, 105:6179-6184.

A modeling study using a biophysically realistic model of the dentate gyrus to show that epileptic synchronization may be supported by the existence of highly anatomically connected excitatory hub cells.

32. Feldt Muldoon S, Soltesz I, Cossart R: Spatially clustered neuronal assemblies comprise the microstructure of synchrony in chronically epileptic networks. Proc Natl Acad Sci U S A 2013, 110:3567-3572.

33. Sabolek HR, Swiercz WB, Lillis KP Cash SS, Huberfeld G, Zhao G, Ste ML, Clemenceau S, Barsh G, Miles R, Staley KJ: A candidate mechanism underlying the variance of interictal spike propagation. J Neurosci 2012, 32:3009-3021.

34. Truccolo W, Donoghue JA, Hochberg LR, Eskandar EN, Madsen JR, Anderson WS, Brown EN, Halgren E, Cash SS: Single-neuron dynamics in human focal epilepsy. Nat Neurosci $2011,14: 635-641$.

35. Keller CJ, Truccolo W, Gale JT, Eskandar E, Thesen T, Carlson C Devinsky O, Kuzniecky R, Doyle WK, Madsen JR, Schomer DL, Mehta AD, Brown EN, Hochberg LR, Ulbert I, Halgren E, Cash SS: Heterogeneous neuronal firing patterns during interictal epileptiform discharges in the human cortex. Brain 2010, 133:1668-1681.

36. Fujiwara-Tsukamoto $Y$, Isomura $Y$, Imanishi M, Ninomiya $T$, Tsukada M, Yanagawa Y, Fukai T, Takada M: Prototypic seizure activity driven by mature hippocampal fast-spiking interneurons. J Neurosci 2010, 30:13679-13689. 